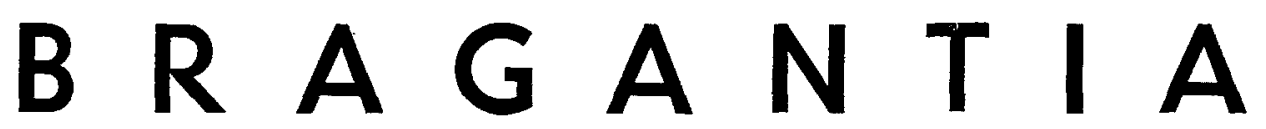

Revista Científica do Instituto Agronômico do Estado de São Paulo

\title{
EFEITOS DA DENSIDADE DE PLANTIO E DA APLICAÇÃO DE CCC, EM ALGODOEIRO ( $\left.{ }^{(}\right)$
}

Carlos A. M. Ferraz, Edivaldo Cia $\left({ }^{2}\right)$, Seção de Algodão, N. P. Sabino ( $\left.{ }^{2}\right)$, José

M. M. Grossi, Seção de Tecnologia de Fibras, A. A. VEIGA, E. E. Tietê, Instituto Agronômico e H. YoshidA, Blemco Importadora e Exportadora Ltda.

\section{SINOPSE}

Foi estudada a associação da aplicação do regulador de crescimento CCC (Chlorocholine Chloride) e densidade de plantio, em algodoeiro. Em Campinas e Sumaré estudou-se o efeito da população de 4, 8 e 16 plantas/metro línear, com e sem CCC. Em Tietê e Piracicaba foram utilizadas 4, 8, 16 e 32 plantas/metro, sencio 16 e 32 com e sem CCC. Aplicou-se a dose de 50 gramas do ingrediente ativo do CCC/ha 50 a 70 dias após a emergência das plantas.

Os resultados mastraram que a aplicação do $\mathrm{CCC}$ retardou o desenvolvimento regetativo das plantas, determinando sensivel diminuiçăo no porte, aumentou a precocidade de abertura dos capulhos, diminuiu a porcentagem de fibra, havendo ainda meIhoria no peso de capulho, peso de sementes e no comprimento das fibras. Náo houve modificação na produçāo de algodāo, como também na uniformidade de comprimento, indice Micronaire, resistência Pressley e maturidade das fibras.

Ocorreu maior produçāo para tratamentos com maior densidade de plantas, comparativamente aos tratamentos com menor densidade. Embora nāo significativo estatisticamente, verificou-se que para maior número de plantas ocorreu aumento no comprimento das fibras e menor peso de capulho.

\section{1 - INTRODUÇÃO}

O excessivo desenvolvimento vegetativo do algodoeiro em determinados locais, com freqüente queda e emaranhamento de plantas, pode determinar prejuízos na colheita e no tipo do algodão produzido, principal-

(1) Trabalho parcialmente apresentado na XXVII Reuniāo Anual da Sociedade Brasileira para - Progresso đa Ciência, Belo Horizonte - MG, 9 a 16 de julho de 1975 . Recebido para publicação em 3 de janeiro de 1977.

(i) Com bolsa de suplementação do C.N.Pq. 
mente para a colheita mecânica, pols as máquinas exigem seja adotado o espaçamento de um metro entre linhas, sendo a eficiência das colhedeiras bastante prejudicada para lavouras com altura média das plantas superior a 1,50m. Para plantas com altura média superior a $1,80 \mathrm{~m}$, o uso de colhedeiras não é recomendado.

Analisando essas situações, vários pesquisadores têm-se dedicado ao estudo do problema, procurando soluções através de hormônios reguladores de crescimento. Através do uso desses produtos, procura-se uma maior uniformidade de altura das plantas, a redução do porte, o aumento da precocidade, sem que ocorram prejuízos ao cotonicultor e às indústrias de beneficiamento e têxtil.

Dos hormônios, Cycocel ou CCC (cloreto de 2-cloro etiltrimetilamônio ou cloreto de clorocolina), tem sido de uso mais intensivo. Thomas (14), aplicando diferentes doses de CCC sobre a variedade de algodão Deltapine 14 (Gossypium hirsutum), reduziu a altura das plantas, a taxa de florescimento e a produção de algodão quando utilizou pulverizaçôes foliares de 25 a 100 ppm de CCC. Silva (11), utilizando a variedade SATU 65 (African Upland Cotton) a $0,6 \mathrm{~m}$ entre linhas e sete plantas por metro, estudou o efeito de dose de CCC em diferentes épocas de aplicação. Os resultados mostraram que o CCC, 38 dias após a emergência foi o mais eficaz na redução do crescimento vegetativo. A aplicação de CCC também reduziu o número de ramos frutíferos e conseqüentemente a piodução.

Zur e colab. (16), testando o efeito do $\mathrm{CCC}$ e do $\mathrm{CMH}, \mathrm{N}$-dimethyl N (B-chloroethyl-hydrazonium) chloride, pulverizaram o algodoeiro antes do florescimento em condições de campo e observaram que esses produtos reduziram consideravelmente a altura das plantas. CCC reduziu também a produção e retardou a maturação. Zur e colab. (17), testando novamente $\mathrm{CMH}$ e $\mathrm{CCC}$, em diferentes doses é épocas de aplicação, observaram efeito sobre a altura, florescimento e produção de algodão. Tollervey (15), trabalhando em Uganda, também encontrou redução na produção e na qualidade do algodão, devida ao tratamento com CCC.

Singh (12) obteve aumento na produção de algodão, utilizando baixas concentrações de CCC. Singh (13), em trabalho realizado na India, aplicou $\mathrm{CCC}$ à base de $40 \mathrm{~g}$ p.a./ha, em variedades americanas e constatou que a produção aumentou de $6,4 \%$ a $22,7 \%$. Lindeley (9) cita que na Grécia é comum a utilização do CCC com a finalidade de reduzir o crescimento apical e aumentar a produção.

Relativamente à qualidade da fibra, Bhatt e Nathian (2) verificaram que as suas propriedades foram afetadas desfavoravelmente quando $o$ CCC foi aplicado em algodoeiro cultivado $\mathrm{em}$ solos encharcados. Por outro lado, Bhatt \& Ramajunan (3) observaram aumento no comprimento e diminuição na finura da fíbra de algodão devido à utilização do CCC.

Com respeito ao espaçamento para o algodoeiro anual, sob condições normais de cultura, recomenda-se para as nossas condições que a distância entre linhas seja $2 / 3$ da altura média das plantas. Righi e colab. (10) relataram que o espaçamento entre linhas não deve ir além de um 
metro, e nas terras fracas deve ficar em torno de $60-70 \mathrm{~cm}$. Esses autores consideram também que se deve deixar de cinco a 10 plantas por metro de fileira, ou seja, 50.000 a 100.000 plantas por hectare para espaçamento de $1 \mathrm{~m}$ entre fileiras. Já Bridge e colab. (4), estudando o efeito da densidade de plantio sobre os caracteres agronômicos e a qualidade da fibra de algodão, mostraram que as maiores produções foram obtidas com a densidade de 114.000 a 121.000 plantas por hectare, ou seja, cinco plantas por cova ou 13 plantas por metro. Também encontraram diferenças sigficativas na porcentagem de fibra, no tamanho das maçãs e índice-de-semente. As características de comprimento, resistência e elongação não foram afetadas, mas o valor do índice Micronaire apresentou tendência a diminuir com o aumento da densidade de plantio.

Hawkins e Peacock (7), estudando o efeito do número de plantas por cova, o espaçamento dentro da fileira e a população de plantas sobre a produção de algodão, encontraram que a mais alta produção foi obtida com cinco plantas por cova, espaçadas de $40 \mathrm{~cm}, 120.412$ plantas por hectare. Os mesmos autores (8) observaram que o tamanho do capulho foi afetado pela densidade de plantio. Burch (5) observou que a resistência da fibra de algodão decresceu com o aumento do número de plantas na linha.

O presente trabalho teve por objetivo estudar o efeito da densidade de plantio associado à aplicação do CCC, nas características agronômicas do algodoeiro e nas propriedades físicas da fibra de algodão.

\section{2 - MATERIAL E METODOS}

Os ensaios foram instalados nos municípios de Tietê, Piracicaba, Campinas e Sumaré, durante os anos agrícolas de 1972/73 a 1975/76, com a finalidade de estudar o efeito da aplicação do produto Cycocel $(2-$ chloroethyltrimethylammonium chloride, ou $\mathrm{CCC}$ ) e da densidade de plantio sobre as características agronômicas do algodoeiro e propriedades físicas da fibra de algodão.

Nos locais dos ensaios de Tietê, Piracicaba e Campinas, o algodoeiro desenvolve-se pouco (até aproximadamente $1,10 \mathrm{~m}$ de altura), enquanto em Sumaré o desenvolvimento é maior.

Foram utilizadas as variedades comerciais de algodoeiro IAC13-1, IAC RM3 e IAC 16, em distribuição no Estado de São Paulo. A semeação foi efetuada na época recomendada, e quinze dias após a emergência foi feito desbaste preliminar. Vinte $e$ cinco dias após a emergência foi acertado o estande inicial, de acordo com o determinado por tratamento (número de plantas por metro linear). Os tratos culturais, adubação e aplicação de inseticidas foram os normalmente recomendados para a cultura na região. Foram estudados dois esquemas de densidade de plantio: Um com 32 plantas por metro linear (Tietê e Piracicaba), e outro com até 16 plantas por metro linear (Campinas e Sumaré).

Os esquemas de densidade de plantio, aplicação do CCC, variedades empregadas, como também anos agrícolas estudados encontram-se nos quadros 1 (Tietê e Piracicaba) e 2 (Campinas e Sumaré). 
QUADRO 1. - Tratamentos e variedades de algodoeiro estudados em Tietê e Piracicaba e respectivos anos agricolas

\begin{tabular}{|c|c|c|c|c|}
\hline $\begin{array}{c}\text { Tratamento } \\
\text { N. }\end{array}$ & $\begin{array}{c}\text { Tietê } \\
\text { (IAC RM3) } \\
\text { 1972/73 e 1973/74 }\end{array}$ & $\begin{array}{l}\text { Tietê } \\
\text { (IAC 16) } \\
1974 / 75\end{array}$ & $\begin{array}{c}\text { Piracicaba } \\
\text { (IAC 13-1) } \\
1974 / 75\end{array}$ & $\begin{array}{c}\text { N. }{ }^{\circ} \text { de plantas } \\
\text { por ha }\end{array}$ \\
\hline 1 & $4 \mathrm{pl} / \mathrm{m}$ & $4 \mathrm{pl} / \mathrm{m}$ & $4 \mathrm{pl} / \mathrm{m}$ & 40.000 \\
\hline 2 & $8 \mathrm{pl} / \mathrm{m}$ & $8 \mathrm{pl} / \mathrm{m}$ & $8 \mathrm{pl} / \mathrm{m}$ & 80.000 \\
\hline 3 & $16 \mathrm{pl} / \mathrm{m}$ & $16 \mathrm{pl} / \mathrm{m}$ & $16 \mathrm{pl} / \mathrm{m}$ & 160.000 \\
\hline 4 & $32 \mathrm{pl} / \mathrm{m}$ & $32 \mathrm{pl} / \mathrm{m}$ & $32 \mathrm{pl} / \mathrm{m}$ & 320.000 \\
\hline 5 & $16 \mathrm{pl} / \mathrm{m}+\mathrm{CCC}$ & $16 \mathrm{pl} / \mathrm{m}+\mathrm{CCC}$ & $16 \mathrm{pl} / \mathrm{m}+\mathrm{CCC}$ & 160.000 \\
\hline 6 & $32 \mathrm{pl} / \mathrm{m}+\mathrm{CCC}$ & $32 \mathrm{pl} / \mathrm{m}+\mathrm{CCC}$ & $32 \mathrm{pl} / \mathrm{m}+\mathrm{CCC}$ & 320.000 \\
\hline
\end{tabular}

QUADRO 2. - Tratamentos e variedades de algodoeiro estudadas em Campinas e Sumaré e respectivos anos agrícolas

\begin{tabular}{c|c|c|c}
\hline $\begin{array}{c}\text { Tratamento } \\
\text { N. }\end{array}$ & $\begin{array}{c}\text { Campinas (IAC RM3) } \\
1972 / 73,1973 / 74 \text { e 1974/75 }\end{array}$ & $\begin{array}{c}\text { Sumare (IAC 13-1) } \\
1973 / 74,1974 / 75 \text { e 1975/76 }\end{array}$ & $\begin{array}{c}\text { N. } .^{\circ} \text { de plantas } \\
\text { por ha }\end{array}$ \\
\hline 1 & $4 \mathrm{pl} / \mathrm{m}$ & $4 \mathrm{pl} / \mathrm{m}$ & 40.000 \\
2 & $8 \mathrm{pl} / \mathrm{m}$ & $8 \mathrm{pl} / \mathrm{m}$ & 80.000 \\
3 & $16 \mathrm{pl} / \mathrm{m}$ & $16 \mathrm{pl} / \mathrm{m}$ & 160.000 \\
4 & $4 \mathrm{pl} / \mathrm{m}+\mathrm{CCC}$ & $4 \mathrm{pl} / \mathrm{m}+\mathrm{CCC}$ & 40.000 \\
5 & $8 \mathrm{pl} / \mathrm{m}+\mathrm{CCC}$ & $8 \mathrm{pl} / \mathrm{m}+\mathrm{CCC}$ & 80.000 \\
6 & $16 \mathrm{pl} / \mathrm{m}+\mathrm{CCC}$ & $16 \mathrm{pl} / \mathrm{m}+\mathrm{CCC}$ & 160.000 \\
\hline
\end{tabular}

O espaçamento entre linhas foi sempre de 1,00 metro, como é exigido para colheita mecânica. As parcelas foram constituídas por quatro linhas de cinco metros de comprimento, sendo consideradas úteis as duas centrais.

O Cycocel foi aplicado através de pulverizador de alta precisão, a 40lb./pol. , uma única vez, 50 a 70 dias após a emergência e na dosagem de $50 \mathrm{~g}$ p.a:/ha.

Nos quadros 1 e 2 constam, inclusive, os diferentes tratamentos com o hormônio regulador de crescimento Cycocel.
A alta densidade de plantas (32 por metro linear) foi estudada em solos onde $o$ algodoeiro apresenta pequeno desenvolvimento vegetativo e é recomendado o espaçamento entre linhas inferior a $1,00 \mathrm{~m}$. Procurou-se a manutenção da população de plantas por área, evitando quebra no rendimento por área de plantio.

Em Tietê, Piracicaba, Sumaré, e Campinas em 1972/73, foi adotado o delineamento estatístico de quadrado latino $6 \times 6$. Esse mesmo número de tratamentos e de repetiçōes foi utilizado para Campinas em 1973/74 e $1974 / 75$, mas neste caso foi adotado 
o delineamento estatístico de blocos ao acaso. Os tratamentos variavam em função da densidade de plantio e da aplicação do Cycocel.

A avaliação estatística dos resultados foi baseada em três análises conjuntas, de acordo com os tratamentos utilizados e desenvolvimento das plantas em cada localidade. Primeiramente, gruparam-se os ensaios instalados em Tietê, nos anos agrícolas de 1972/73, 1973/74 e 1974/75, e efetuado em Piracicaba no ano agrícola de 1974/75. A segunda análise foi feita com ensaios instalados em Campinas nos anos agrícolas de $1972 / 73,1973 / 74$ e $1974 / 75$, e a terceira com ensaios instalados em Sumaré nos anos agrícolas de 1973/ $74,1974 / 75$ e $1975 / 76$.

Para a comparação entre as médias dos tratamentos foi utilizado o teste de Tukey, a 5\% de probabilidade. Para algumas características com valores $F$ sígnificativos, nos ensaios de Campinas e Sumaré foi aplicado o teste de Scheffé, a $5 \%$ de probabilidade, comparando-se os tratamentos com e sem CCC.

As características agronômicas estudadas foram: produção, porcentagem de fibra, peso de cem sementes, peso de capulho, precocidade e altura de plantas. A precocidade foi obtida pela relação entre o peso da primeira colheita e total de produção de cada parcela. A altura das plantas foi determinada por ocasião da colheita do algodão produzido.

Os estudos de laboratório foram baseados em amostras de 20 capulhos tirados do terço médio das plantas, sendo feitas determinações do comprimento, uniformidade de comprimento, resistência Pressley e índice
Micronaire, segundo as normas apresentadas pela Sociedade Americana para testes de Materiais (1). Determinou-se também a maturidade das fibras, segundo o método proposto por Gridi-Papp \& Sabino (6).

\section{3 - RESULTADOS}

As médias dos resultados das características econômicas do algodoeiro, obtidas em Tietê e Piracicaba, referentes à primeira análise conjunta, como também os valores do teste $F$, dos coeficientes de variação e do teste de Tukey, encontram-se no quadro 3. Nesse quadro, como também nos posteriores, são ainda apresentadas as médias para tratamentos sem $\mathrm{CCC} \mathrm{e}$ com CCC.

Observa-se no quadro 3 , que $F$ foi significativo para as seguintes características: altura, produção, precocidade, porcentagem de fibra, peso de 100 sementes e comprimento de fibra. As características de peso de capulho, uniformidade de comprimento, índice Micronaire, resistência Pressley e maturidade não apresentaram F significativo.

A altura das plantas diminuiu significativamente com o aumento da densidade de plantio, e também com a aplicação do $\mathrm{CCC}$. A produção apresentou acréscimo com o aumento da densidade de plantio, sendo maior para o tratamento com 16 plantas/ metro, não havendo efeito para o CCC. A precocidade foi influenciada significativamente no tratamento de 32 plantas/metro, pela aplicação do CCC. A porcentagem de fibra diminuiu significativamente com a aplicação do $\mathrm{CCC}$, ocorrendo o inverso com respeito ao peso de 100 semen- 


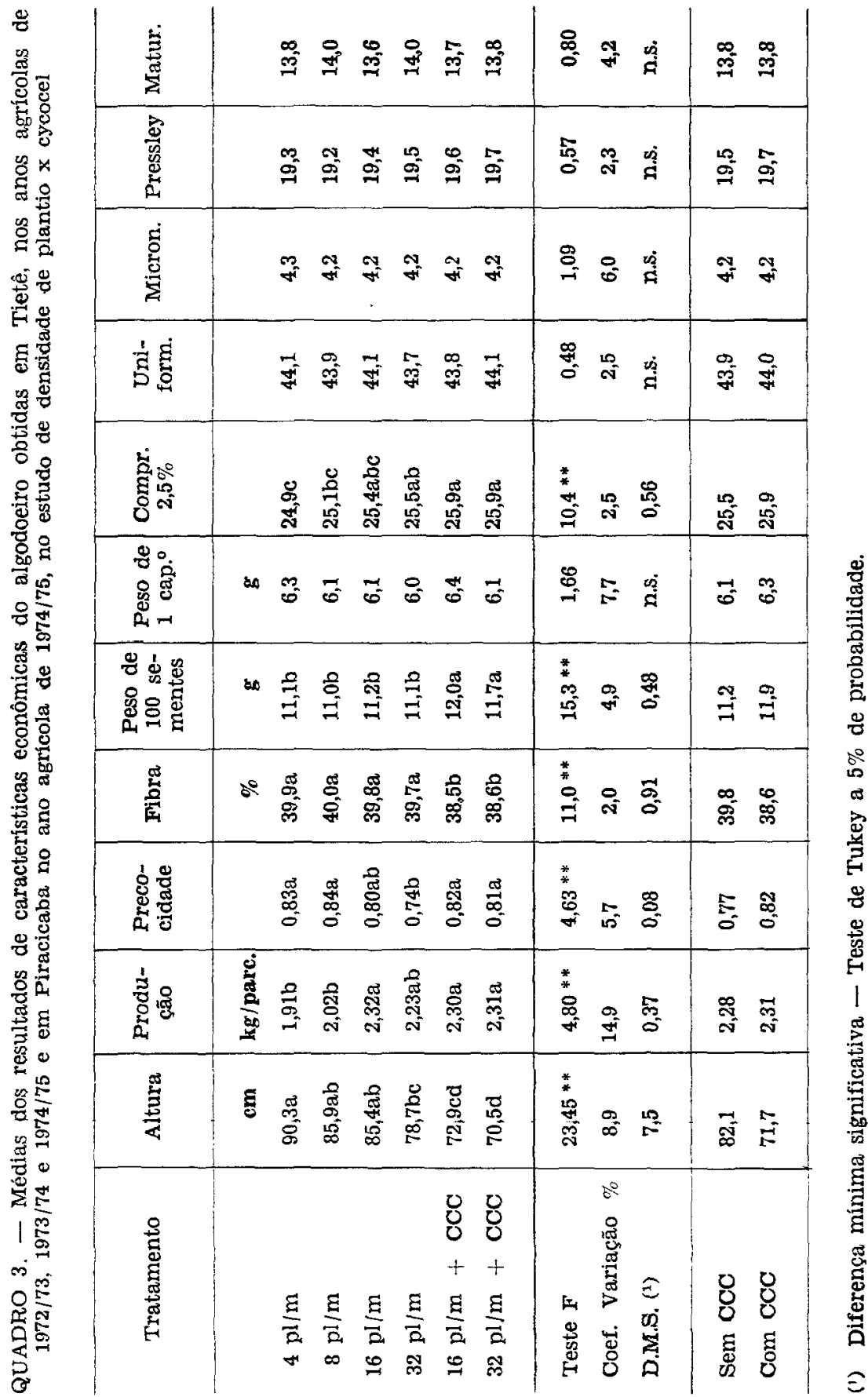


tes. Embora não significativo, o comprimento de fibra foi maior com a aplicação do CCC.

Em geral, a primeira análise conjunta mostrou que a aplicação do CCC limitou a altura das plantas e diminuiu a porcentagem de fibra $\mathrm{e}$ aumentou a precocidade e peso de 100 sementes, sem afetar as características de produção, peso de um capulho, uniformidade de comprimento, índice Micronaire, resistência Pressley e maturidade. Por outro lado, o aumento da densidade de plantio limitou a altura das plantas e diminuiu a precocidade, e aumentou a produção e comprimento de fibras sem afetar as outras características.

As médias dos resultados das características econômicas do algodoeiro, obtidas em Campinas, referente à segunda análise conjunta, como também os valores de $\mathrm{F}$, dos coeficientes de variação e dos testes de Tukey e Scheffé, encontram-se no quadro 4 .

Observa-se no quadro 4 que $F$ foi significativo para as seguintes características: altura, produção, precocidade, porcentagem de fibra, peso de 100 sementes, peso de um capulho e comprimento de fibra. As características de uniformidade de comprimento, índice Micronaire, resistência Pressley e maturidade não apresentaram $F$ significativo.

A altura das plantas foi significativamente menor com a aplicação do CCC. A produção foi influenciada pela densidade de plantio, sendo melhor para o tratamento de oito plantas/metro. A densidade de plantio influenciou significativamente a precocidade, sendo melhor para os tratamentos com menos plantas. Embonão significativa, a precocidade foi acelerada pela aplicação do $\mathrm{CCC}$. $\mathrm{O}$ tratamento de 4 plantas/metro apresentou porcentagem de fibra significativamente maior quando não se utilizou o CCC, ocorrendo o inverso para peso de um capulho.

Em média, a segunđa análise conjunta mostrou que com a aplicação do CCC houve diminuição da altura das plantas e da porcentagem de fibra e notou-se tendência de aumento, embora não significativamente, da precocidade, do peso de 100 sementes, peso de capulho e comprimento de fibra, sem que fossem afetadas as outras características estudadas. A maior produção foi registrada para o tratamento com 8 plantas/metro em relação ao de 4 plantas/metro, sem aplicação do CCC. A precocidade foi maior para o tratamento com 4 plantas/metro, em relação a 16 plantas/metro. O teste de Scheffé mostrou significância somente para a característica de altura das plantas.

As médias dos resultados das características econômicas do algodoeiro, obtidas em Sumaré, referentes à terceira análise conjunta, como também os valores $F$, os coeficientes de variação e os testes de Tukey e Scheffé, encontram-se no quadro 5.

Observa-se pelo quadro 5 que $\mathrm{F}$ foi significativo para as seguintes características: altura, precocidade, porcentagem de fibra, peso de 100 sementes, peso de um capulho e comprimento de fibra. As características de produção, uniformidade de comprimento, índice Micronaire, resistência Pressley e maturidade não apresentaram $\mathrm{F}$ significativo.

A altura das plantas e porcentagem de fibras foram menores significativamente com a aplicação do CCC, 


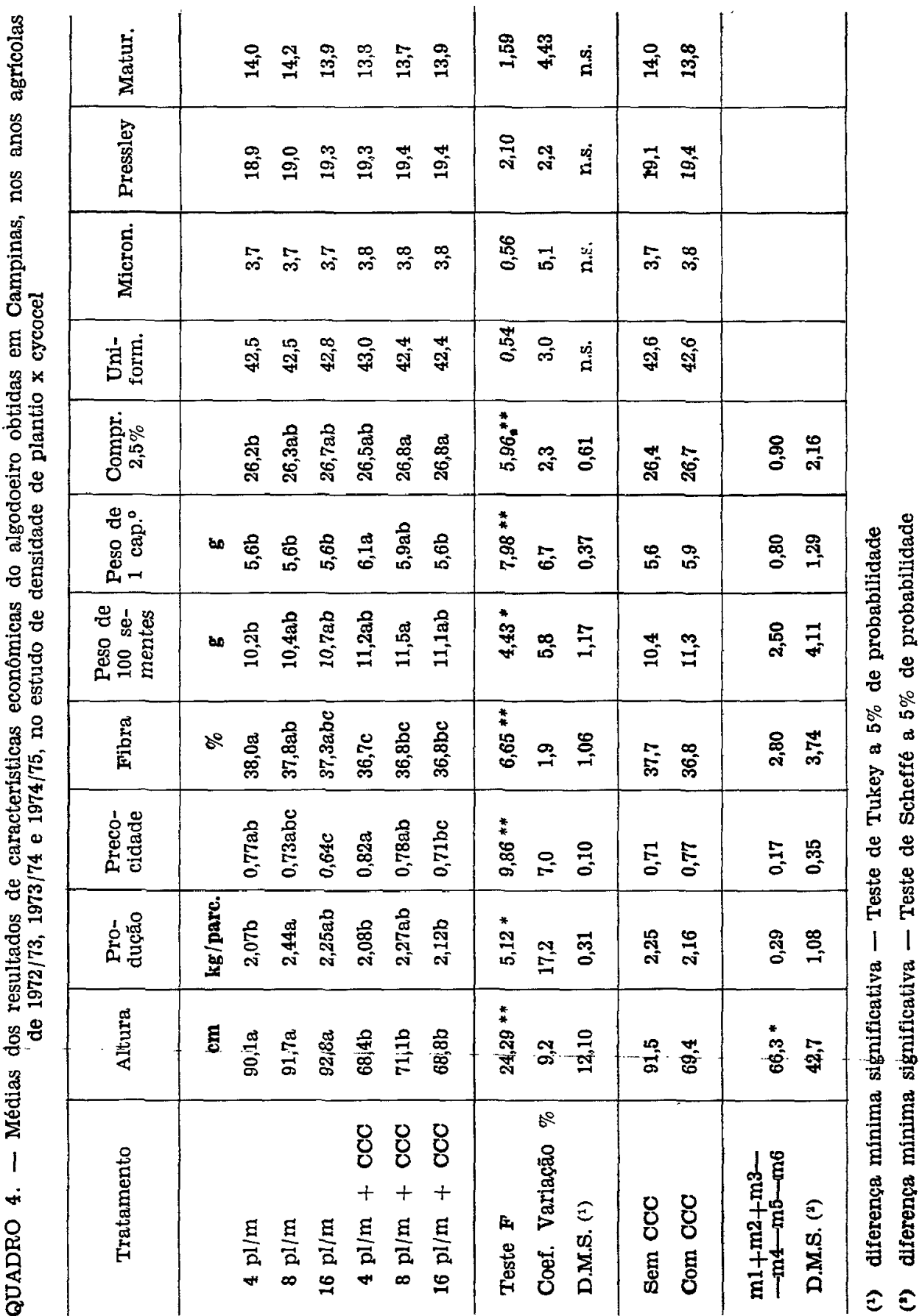




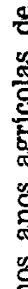

⿷匚

क.

孚 $x$

융웅

늠

ष्ष

तั

월

농

옹

๘。

要家

要究

进票

赵。

웅

옹

䒿

\begin{tabular}{|c|c|c|c|c|c|c|c|c|}
\hline $\begin{array}{l}\text { 荧 } \\
\text { 营 }\end{array}$ & $\stackrel{\infty}{=}$ & $\stackrel{0}{\dddot{m}} \stackrel{2}{9}$ & & בֶ: & స્త్ & 点 究 & 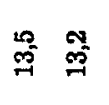 & \\
\hline 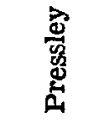 & $\stackrel{\circ}{2}$ & $\stackrel{5}{\circ}$ & & 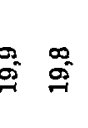 & $\stackrel{\infty}{\sigma}$ & 111 & 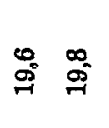 & \\
\hline 密 & $\neq$ & $\approx ?$ & zi & $\vec{j}$ & $\stackrel{*}{*}$ & $\stackrel{9}{\Rightarrow} \stackrel{4}{q}$ & * * & \\
\hline 它莒 & $\underset{*}{*}$ & सं & $\infty$ & $\underset{F}{*}$ & $\$$ & 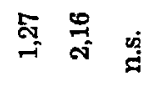 & $\stackrel{\infty}{\sharp}$ & \\
\hline $\begin{array}{l}\text { 总品 } \\
\text { 品 }\end{array}$ & 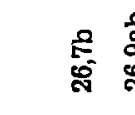 & 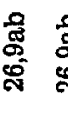 & & సึ & 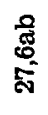 & 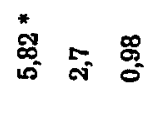 & 查 & 워 옳 \\
\hline 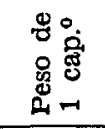 & 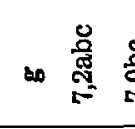 & $\stackrel{\circ}{\circ}$ & & 总 & 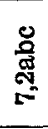 & 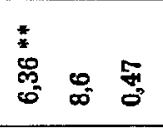 & $\stackrel{9}{=}$ & 요 둗 \\
\hline 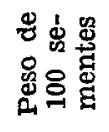 & 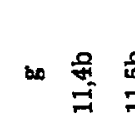 & $\begin{array}{l}\text { दी } \\
\Rightarrow \\
=\end{array}$ & & 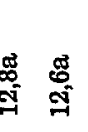 & ณึ & $\begin{array}{l}* \\
\mathbb{0} \\
\stackrel{7}{*} \neq\end{array}$ & $\stackrel{0}{\Rightarrow} \stackrel{0}{=}$ & 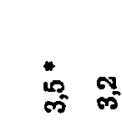 \\
\hline 茞 & s 용 & 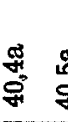 & 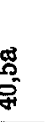 & 尽 尽 & 艿 & 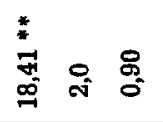 & $\stackrel{0}{\overline{8}} \overrightarrow{8}$ & F \\
\hline 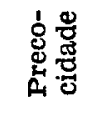 & 存 & 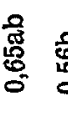 & & $\underset{8}{8}$ & 兽 & 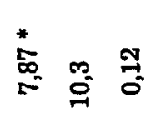 & : & 密 苟 \\
\hline 起密 & 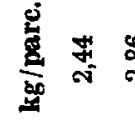 & 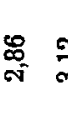 & sy & 至 & 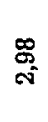 & के & 点 & 긍 : \\
\hline 莺 & छ & 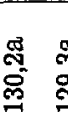 & בֶ: & $\begin{array}{l}80 \\
80 \\
80\end{array}$ & $\begin{array}{l}\text { 응 } \\
\text { : } \\
0\end{array}$ & 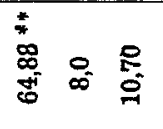 & 孚 & $\stackrel{*}{*}$ \\
\hline 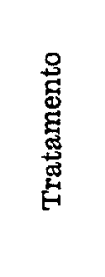 & $\begin{array}{l}\mathrm{g} \\
\frac{5}{2} \\
+1 \\
+1\end{array}$ & $\begin{array}{ll}\mathbb{E} \\
\frac{E}{2} \\
\infty \\
\infty\end{array}$ & $\frac{5}{2}$ & 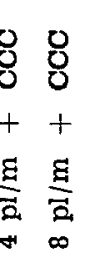 & $\begin{array}{l}0 \\
0 \\
+ \\
8 \\
\vdots \\
0 \\
0\end{array}$ & 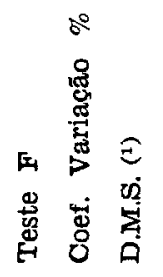 & \begin{tabular}{ll}
0 & 0 \\
0 & 0 \\
0 & 0 \\
$\Xi$ & $\Xi$ \\
\hdashline & 0
\end{tabular} & 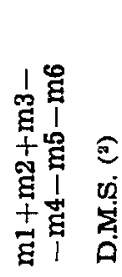 \\
\hline
\end{tabular}

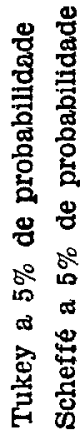

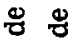

范 F

䞞

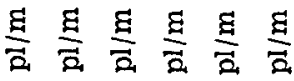

$>$ म

क्ष

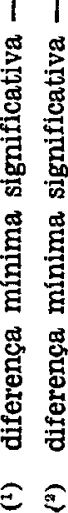


não havendo diferença para densidade de plantio. $O$ peso de 100 sementes aumentou significativamente com a aplicação do CCC, sem ocorrer diferença com a densidade de plantio. $O$ teste de Scheffé mostrou significância para as características de altura, precocidade, porcentagem de fibra $\mathrm{e}$ peso de 100 sementes.

Em média, a terceira análise conjunta mostrou que a aplicação do CCC limitou a altura das plantas, diminuiu a porcentagem de fibra e determinou aumento da precocidade e peso de 100 sementes. Embora não significativamente, houve tendência para aumento no peso de um capulho e comprimento da fibra, sem que as outras característcias estudadas fossem afetadas. Com relação à densidade de plantio, os resultados mostraram que houve aumento de produção para o tratamento de maior densidade (16 plantas/metro) embora não ocorressem diferenças significativas entre os tratamentos.

\section{4 - DISCUSSÃO}

Os resultados das análises conjuntas mostraram efeitos significativos para emprego de $\mathrm{CCC}$, como para diferentes densidades de plantio.

A altura das plantas foi a característica mais influenciada pelos tratamentos. O CCC reduziu sempre a altura das plantas, sendo a redução maior nos locais onde as plantas se desenvolveram mais. Nesse caso, a redução foi em torno de $30 \%$. O termo redução é empregado para indicar que a planta deixou de crescer ou que o seu crescimento foi menor em relação às plantas que não receberam o CCC. O fato de ocorrer a limitação da altura pelo emprego do CCC já foi observado por outros autores como Silva (11), Thomas (14), Tollervey (15) e Zur e colab. (16, 17). A limitação da altura das plantas é muito importante onde o desenvolvimento normal das mesmas ultrapassa 2 1,50 metro, pois a colheita do algodão torna-se mais ònerosa e difícil, principalmente quando se empregam colhedeiras mecânicas.

O produto regulador de crescimento CCC aumentou significativamente a precocidade, o peso de 100 sementes, o peso de um capulho e o comprimento da fibra, e diminuiu significativamente a porcentagem de fibra. Para o algodoeiro, várias características econômicas são correlacionadas entre si. No presente caso, houve o aumento de peso de 100 sementes, peso de um capulho e comprimento da fibra, em detrimento da diminuição da porcentagem de fibra. Com respeito à precocidade, os resultados deste trabalho contradizem os obtidos por Zur e colab. (16, 17), onde esses autores obtiveram atraso na maturação do algodão com a aplicação do CCC.

Bhatt \& Nathan (2) e Tollervey (15) encontraram efeitos prejudiciais para a qualidade de algodão, enquanto Bhatt \& Ramanujan (3) encontraram aumento no comprimento, devido ao $\mathrm{CCC}$. Os resultados deste trababathe concordaram com os últimos autores, pois foram obtidos aumentos significativos no comprimento da fibra de algodão.

Embora outros autores como Silva (11), Thomaz (14), Tollervey (15) e Zur e colab. (16, 17) encontrassem resultados inversos, os experimentos mostraram que a produção de 
algodão, em média, não foi afetada pelo CCC. Esse fato mostra a possibilidade de os cotonicultores utilizarem o CCC como regulador do crescimento das plantas, sem prejuízo na produção.

Com respeito à densidade de p'antio, foi observado na análise conjunta de Tietê e Piracicaba que as plantas se desenvolveram menos nos tratamentos de maior densidade. Por outro lado, em Campinas e Sumaré não houve esse efeito, embora o número máximo tenha sido de 16 plantas/metro. A precocidade também diminuiu em função da altura das p?antas. Isto pode ser explicado pela concorrência entre as plantas nas linhas.

No caso de produção, houve aumento significativo para maior densidade em Tietê e Piracicaba, onde a maior produção ocorreu com o tratamento de 16 plantas/metro. Em Campinas, embora com resultado não significativo, a maior produção foi obtida com 8 plantas/metro e em Sumaré com 16 plantas/metro. $\mathrm{O}$ ganho em produção quando se aumenta o número de plantas por área já foi observado por outros pesquisadores como Bridge e colab. (4) e Hawkins e Peacock (7). Como foi utilizado um espaçamento único de um metro entre linhas, o aumento da produção para as maiores densidades poderia ser explicado com o acerto do número de plantas por área, uma vez que o espaçamento recomendado entre linhas é de $2 / 3$ da altura média das plantas (10). Este fato tem também grande importância para a correção do espaçamento para uso de colhedeiras mecânicas, que exigem espaçamento de $1,00 \mathrm{~m}$ entre linhas, onde o algodoeiro normalmente é plantado a uma distância menor que $1,00 \mathrm{~m}$, entre linhas.

Bridge e colab. (4), Burch (5) e Hawkins e Peacock $(\mathbf{7}, \mathbf{8})$ encontraram efeitos significativos negativos em função da maior densidade de plantio, para as seguintes características: porcentagem de fibra, tamanho de maçãs, índice de sementes, índice Micronaire e resistência da fibra. Neste trabalho foi observada uma tendência dos tratamentos com maior número de plantas para apresentarem maior comprimento de fibra e menor peso de um capulho.

\section{5 - CONCLUSÕES}

Os resultados obtidos neste trabalho permitem concluir:

a) O emprego de CCC limitou o crescimento das plantas, determinou maior precocidade na abertura dos capulhos, sem que ocorressem perdas na produção. Ocorreu, ainda, diminuição na porcentagem de fibra e ganho no peso de um capulho, peso de 100 sementes e comprimento de fibra, sem que as demais características fossem afetadas.

b) Houve aumento na produção para os tratamentos com maior número de plantas por metro e tendência para maior comprimento de fibra e menor peso de capulho. As demais características não foram afetadas. 


\section{INFLUENCE OF GROWTH LIMITANT GHEMICAL GCC AND POPULATION DENSITY ON COTTON}

\section{SUMMARY}

Excessive growth of the cotton plants Gossypium hirsutum L. in some regions causes laying and deficient luminosity, and a consequent lowering in yield and type of cotton. Such problems are specially serious in the case of mechanical picking where between-row spacing is fixed at about 40 inches.

Combined effects of the use of growth limitant chemical CCC (Chlorocholine Chloride) and population density were studied in 1972/73 and 1975/76 in four localities of the State of São Paulo. Near Campinas and Sumaré, the effects of 4, 8 and 16 plants/meter (respectively $40,000,80,000$ and 160,000 plants/ha) with and without CCC were studied. At Tietê and Piracicaba, 4, 8, 16 and 32 plants/meter were used (respectively 40,000, $80,000,160,000$ and 320,000 plants/ha). Only the treatments of 16 and 32 plants/meter were combined with CCC application. The concentration of CCC used was of $50 \mathrm{~g}$ a.j./ha applied from 50 to 70 days after emergence of the plants.

The results showed that CCC limited the growth of plants, increased earliness of yield, decreased lint percentage and increased fiber length, boll and seed weight. There was no effect observed on cotton yield, fiber length uniformity, Micronaire, Pressely and fiber maturity.

Higher density resulted in higher yield. High density also caused better length of fiber and lower boll weight.

\section{LITERATURA CITADA}

1. ASTM Standards on Textile Materials. Philadelphia, American Society for Testing and Materials, $1963.1005 p$.

2. BHATT, J. G. \& NATHAN, A. R. S. Effect of Cycocel, Phosfon and B-nine on growth and yield of cotton (G. hirsutum). J. Pl. Physiol. 13:225-230, 1968.

3. - \& RAMANUJAN, T. Effect of cycocel on yield, chlrophyll content and fiber properties on lint of MCU cotton. Ind. J. PI. Physiol. 13, 1970.

4. BRIDGE, R. R.; MERIDITH, W. R. \& CHISM, J. F. Influence of planting method and plant population on cotton. Agron. J. 65:104-109, 1973.

5. BURCH, T. A. Effects of plant spacing on varieties, yield, fibre properties and growth habits of cotton. Diss. Abst. 1544B, 1969. 29p.

6. GRIDI-PAPP, I. L. \& SABINO, N. P. Maturidade da fibra de algodão determinada por processo óptico - Método do Fibrógrafo Digital. Rev. Bras. de Tec. 3:99-106, 1972 .

7. HAWKINS, B. S. \& PEACOCK, H. A. Yield response of Upland cotton (Gossypium hirsutum L.) to several spacing arrangements. Agron. J. 62:578-580, 1970.

8. \& I I - Influence of row width population density on yield and fibre characteristics of cotton. Agron. J. 65:47-51, 1973.

9. LINDLEY, C. D. A review of some practical uses of cycocel plant growth regulant. PANS 19(1):87-92, 1973.

10. RIGHI, N. R.; FERRAZ, C. A. M. \& CORRĖA, D. M. Cultura e adubação do algodoeiro. VII - Cultura. São Paulo, Instituto Brasileiro da Potassa Experimentação e Pesquisas, 1965. p.235-317. 
11. SILVA, W. H. Some effects of the growth retardant chemical CCC on cotton in Uganda. Cott. Gr. Rev. 48:131-135, 1971.

12. SINGH, S. Revolution in cotton yield with CCC. Indian Farming 20(8):5-6, 1970.

13. $21(4): 28-33,1971$.

14. THOMAS, R. O. Effects of application timing and concentration of 2-chloroethyl trimethylammonium chloride on plant size fruiting responses of cotton. Crop. Sci. 4:403-405, 1964.

15. TOLLERVEY, F. E. Physiology of the cotton plant: Cotton Research Corporation Summer Meeting, 1970. Cott. Grow. Rev. 47:245-256, 1970.

16. ZUR, M.; MARANI, A. \& CARMELI, R. Effect of CMH (N-dimethyl-N) (B-chloroethyl - hydrazonium chloride) as compared with that of CCC (2-chloroethyl-trimethylammonium chloride) on height, earliness and yield of cotton. Israel J. Agric. Res, 20:133-134, 1970.

17.

\& KARADAVID, B. Effect of growth retardants CCC and CMH on cotton. Cott. Grow. Rev. 49:250-257, 1972. 